Article

\title{
Fabrication and Characteristics of a Three-Axis Accelerometer with Double L-Shaped Beams
}

\author{
Ying Wang, Xiaofeng Zhao * and Dianzhong Wen \\ The Key Laboratory of Electronics Engineering, College of Heilongjiang Province, Heilongjiang University, \\ Harbin 150080, China; 2181212@s.hlju.edu.cn (Y.W.); wendianzhong@hlju.edu.cn (D.W.) \\ * Correspondence: zhaoxiaofeng@hlju.edu.cn; Tel.: +86-451-8660-8457
}

Received: 17 February 2020; Accepted: 20 March 2020; Published: 24 March 2020

\begin{abstract}
A three-axis accelerometer with a double L-shaped beams structure was designed and fabricated in this paper, consisting of a supporting body, four double L-shaped beams and intermediate double beams connected to two mass blocks. When applying acceleration to the accelerometer chip, according to the output voltage changes of three Wheatstone bridges constituted by twelve piezoresistors on the roots of the beams, the corresponding acceleration along three axes can be measured based on the elastic force theory and piezoresistive effect. To improve the characteristics of the three-axis accelerometer, we simulated how the width of the intermediate double beams affected the characteristics. Through optimizing the structure size, six chips with different widths of intermediate double beams were fabricated on silicon-on-insulator (SOI) wafers using micro-electromechanical systems (MEMS) technology and were packaged on printed circuit boards (PCB) by using an electrostatic bonding process and inner lead bonding technology. At room temperature and $V_{\mathrm{DD}}=5.0 \mathrm{~V}$, the resulting accelerometer with an optimized size $(w=500 \mu \mathrm{m})$ realized sensitivities of $0.302 \mathrm{mV} / \mathrm{g}$, $0.235 \mathrm{mV} / \mathrm{g}$ and $0.347 \mathrm{mV} / \mathrm{g}$ along three axes, with a low cross-axis sensitivity. This result provides a new strategy to further improve the characteristics of the three-axis accelerometer.
\end{abstract}

Keywords: three-axis accelerometer; double L-shaped beam; MEMS technology; cross-axis sensitivity

\section{Introduction}

In recent years, with the rapid development of semiconductor technology, accelerometers have been widely used in many different fields, mainly including vibration detection [1], consumer electronics [2], medical treatment [3,4], vibration detection of wind turbines [5], navigation [6,7], automobile safety [8], and so on. Currently, although accelerometers have met the needs of real life, they are still facing the challenge of a wide measurement range, high accuracy, etc. Thus, high performance accelerometers have stimulated a particular attention from researchers, providing an opportunity to improve the characteristics of the accelerometer, making it possible to overcome the above mentioned limitation. For example, Sankar et al. proposed a quad beam silicon piezoresistive $z$-axis accelerometer with a very low cross-axis sensitivity of $0.316 \mu \mathrm{V} / \mathrm{V} \cdot \mathrm{g}$ for an in-plane acceleration in 2013 [9]. After that, Han et al. presented a piezoresistive accelerometer with a low cross-axis sensitivity based on masked-maskless wet etching, realizing sensitivities of $0.069 \mathrm{~V} / \mathrm{V} \cdot \mathrm{g}, 0.034 \mathrm{~V} / \mathrm{V} \cdot \mathrm{g}$ and $4.15 \mathrm{~V} / \mathrm{V} \cdot \mathrm{g}$ along the $x$-axis, $y$-axis and $z$-axis, and cross-axis sensitivities of $1.67 \%$ and $0.82 \%$ along the $x$-axis and $y$-axis, respectively [10]. Recently, Takahashi et al. reported an angular acceleration sensor composed of liquid ring channels and piezoresistive cantilevers as the sensing element, achieving an angular acceleration resolution of $0.01 \mathrm{rad} / \mathrm{s}^{2}$ in the range of $0.1-100 \mathrm{~Hz}$ on a target axis about 100 times higher than that of the other axes, a high sensitivity and a low cross-interference [11]. Nowadays, the accelerometer can be divided into several types, mainly including piezoresistive, capacitive [12], piezoelectric [13], etc. The piezoresistive accelerometer, when compared with the ones having other structures, exhibits many 
excellent properties, such as a fast response, easy signal processing and miniaturization, etc. However, the piezoresistive accelerometers are also facing challenges with respect to improving measurement consistency and reducing cross-interference, which have limited their further development.

To overcome the above obstacles, a piezoresistive three-axis accelerometer with double L-shaped beams was designed and fabricated on a silicon-on-insulator (SOI) wafer using MEMS technology in this study. Using the double L-shaped beams concentrated the stress distribution under the actions of $a_{x}$ and $a_{y}$. Through a simulation to investigate how the width of the intermediate double beams affects the characteristics of an accelerometer, the structure size of the intermediate double beams was optimized. The test results indicate that it is possible to achieve the measurement of acceleration along three axes and reduce the cross-axis sensitivity using the resulting accelerometer. The study on the effects of intermediate double beams' structure sizes on the sensitivity of the proposed accelerometer provides a possibility to further improve the performance of three-axis accelerometers.

\section{Basic Structure and Working Principle}

\subsection{Basic Structure}

The three-axis accelerometer is composed of an elastic element and a sensitive element, where the elastic element consists of four double L-shaped beams, intermediate double beams and two mass blocks, and the sensitive element has twelve piezoresistors. The basic structure of the three-axis accelerometer with double L-shaped beams is shown in Figure 1, including a top view, bottom views, as well as a cross-section view along $\mathrm{AA}^{\prime}$. As shown in Figure $1 \mathrm{a}, \mathrm{b}$, the two mass blocks $\left(\mathrm{m}_{1}\right.$ and $\left.\mathrm{m}_{2}\right)$ are first connected by the intermediate double beams $\left(1_{z 1}\right.$ and $\left.l_{z 2}\right)$ and are then connected to the support body by four double L-shaped beams $\left(1_{x 1}, 1_{x 2}, 1_{x 3}, 1_{x 4}, 1_{y 1}, 1_{y 2}, 1_{y 3}\right.$ and $1_{y 4}$, ) on a SOI wafer. Figure $1 c$ gives the cross-section view along $\mathrm{AA}^{\prime}$ for the three-axis accelerometer, in which the thicknesses of the intermediate double beams are the same as those of the four double L-shaped beams.

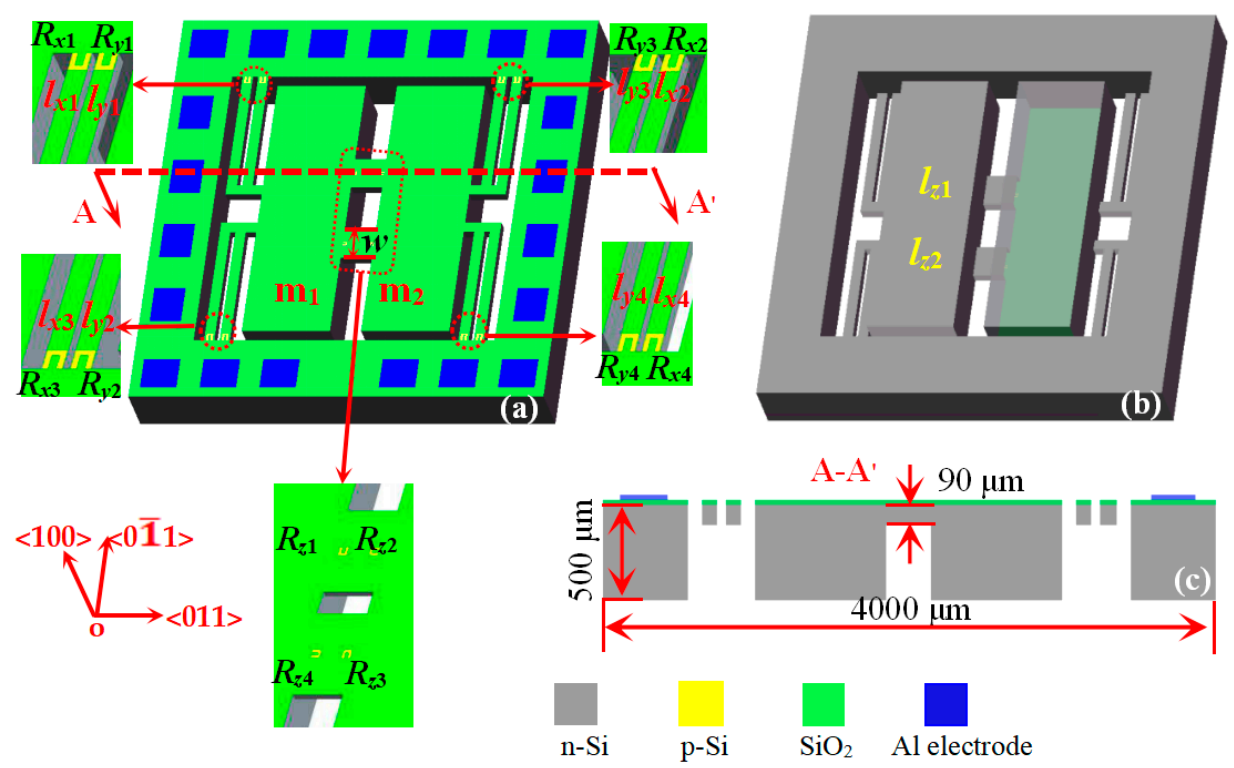

Figure 1. The basic structure of the three-axis accelerometer with double L-shaped beams: (a) top view; (b) bottom view; and (c) cross-section view along $\mathrm{AA}^{\prime}$.

As seen in Figure 1, the twelve piezoresistors $\left(R_{x 1}, R_{x 2}, R_{x 3}, R_{x 4}, R_{y 1}, R_{y 2}, R_{y 3}, R_{y 4}, R_{z 1}, R_{z 2}, R_{z 3}\right.$ and $R_{z 4}$ ) were designed on the roots of four double L-shaped beams and intermediate double beams, where the four piezoresistors $\left(R_{x 1}, R_{x 2}, R_{x 3}\right.$ and $\left.R_{x 4}\right)$ along the $<0 \overline{1} 1>$ orientation are fabricated on the four L-shaped single beams $\left(1_{x 1}, 1_{x 2}, 1_{x 3}\right.$ and $\left.l_{x 4}\right)$ to form a Wheatstone bridge $\left(W_{1}\right)$. Meanwhile, the four piezoresistors $\left(R_{y 1}, R_{y_{2}}, R_{y_{3}}\right.$ and $\left.R_{y 4}\right)$ along the $<0 \overline{1} 1>$ orientation are fabricated on the four L-shaped 
single beams $\left(l_{y 1}, l_{y 2}, l_{y 3}\right.$ and $\left.l_{y 4}\right)$ to constitute a Wheatstone bridge $\left(W_{2}\right)$, and the other piezoresistors $\left(R_{z 1}\right.$, $R_{z 2}, R_{z 3}$ and $\left.R_{z 4}\right)$ along the $<0 \overline{1} 1>$ and $<011>$ orientations are respectively fabricated on the intermediate double beams $\left(l_{z 1}\right.$ and $\left.l_{z 2}\right)$ to form a Wheatstone bridge $\left(W_{3}\right)$. In order to study the effect of the width $(w)$ of the intermediate double beams $\left(l_{z 1}\right.$ and $\left.l_{z 2}\right)$ on the characteristics of the proposed accelerometer, six chip sizes combined with the simulation in Section 3 of this study are shown in Table 1.

Table 1. The parameters of the six chips with different $w$ values.

\begin{tabular}{ccccccc}
\hline Type & AS-1 & AS-2 & AS-3 & AS-4 & AS-5 & AS-6 \\
\hline$w(\mu \mathrm{m})$ & 250 & 300 & 350 & 400 & 450 & 500 \\
\hline
\end{tabular}

\subsection{Sensitivity Analysis}

Figure 2 shows the equivalent circuit of the proposed three-axis accelerometer under the actions of $a_{x}, a_{y}$ and $a_{z}$. When the supply voltage is $V_{\mathrm{DD}}$ and no acceleration is applied to the chip, the output voltages of three Wheatstone bridges consisting of twelve piezoresistors are zero due to the fact that the resistances of the twelve piezoresistors are the same under ideal conditions. When exerting acceleration $a_{x}$ along the $x$-axis to the chip, the four double L-shaped beams will produce deformations. According to the piezoresistive effect of the semiconductor material, the four piezoresistors of $R_{x 1}, R_{x 2}, R_{x 3}$ and $R_{x 4}$ on the roots of $1_{x 1}, 1_{x 2}, 1_{x 3}$ and $1_{x 4}$ are changed, i.e., the reducing of $R_{x 1}$ and $R_{x 3}$ attributed to the inducted compressive stress acted on the roots of $1_{x 1}$ and $1_{x 3}$, while in contrast the increases of $R_{x 2}$ and $R_{x 4}$ caused by the tensile stress acted on the roots of $1_{x 2}$ and $l_{x 4}$. The absolute variations of four piezoresistors are equal in ideal conditions, named as $\Delta R_{x}$. Due to the fact that the output voltages $V_{x 1}$ and $V_{x 2}$ of $\mathrm{W}_{1}$ are relative to the acceleration along the $x$-axis, it is possible to realize the measurement of $a_{x}$. When applying the acceleration $a_{y}$ along the $y$-axis to the chip, the four double L-shaped beams will show deformations, and $R_{y 1}, R_{y 2}, R_{y 3}$ and $R_{y 4}$ located on the roots of $1_{y 1}, 1_{y 2}, l_{y 3}$ and $l_{y 4}$ are changed, resulting in the reduction of $R_{y 1}$ and $R_{y 3}$ due to the induced compressive stress action on the roots of $l_{y 1}$ and $l_{y 3}$, as well as in the increase of $R_{x 2}$ and $R_{x 4}$ caused by the tensile stress acting on the roots of $l_{y 2}$ and $l_{y 4}$. The same absolute variations of four piezoresistors in ideal conditions are regarded as $\Delta R_{y}$. The change of the output voltages $V_{y 1}$ and $V_{y 2}$ of $\mathrm{W}_{2}$ under the action of $a_{y}$ makes it possible to achieve the measurement of $a_{y}$. In addition, when exerting the acceleration $a_{z}$ along the $z$-axis to the chip, the intermediate double beams $l_{z 1}$ and $l_{z 2}$ will produce a deformation along the $z$-axis, and $R_{z 1}$, $R_{z 2}, R_{z 3}$ and $R_{z 4}$ on the roots of $l_{z 1}$ and $l_{z 2}$ are changed, indicating that $R_{z 1}$ and $R_{z 3}$ are influenced by compressive stress, leading to the decreases of $R_{z 1}$ and $R_{z 3}$ and the increases of $R_{z 2}$ and $R_{z 4}$. Similarly, the same absolute variations of four piezoresistors in ideal conditions are regarded as $\Delta R_{z}$. The changes of the output voltages $V_{z 1}$ and $V_{z 2}$ of $W_{3}$ under the action of $a_{z}$ contribute to the measurement of $a_{z}$.

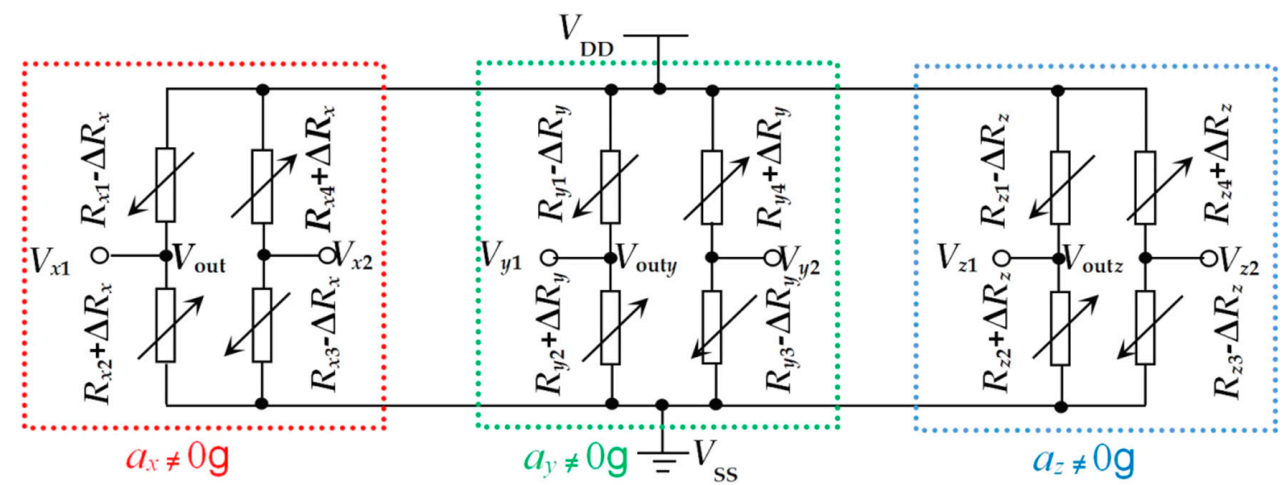

Figure 2. The equivalent circuit of the three-axis accelerometer under the action of $a_{x}, a_{y}$ and $a_{z}$.

When applying acceleration to the chip, the deformations of the proposed accelerometer beams would cause the variations of piezoresistors, where $\Delta R_{x}, \Delta R_{y}, \Delta R_{z}$ are the changes of piezoresistors under the actions of $a_{x}, a_{y}$ and $a_{z}$, respectively. 
In an ideal case, $R_{x 1}=R_{x 2}=R_{x 3}=R_{x 4}=R_{x 0}, R_{y 1}=R_{y 2}=R_{y 3}=R_{y 4}=R_{y 0}, R_{z 1}=R_{z 2}=R_{z 3}=R_{z 4}=R_{z 0}$. In view of the equivalent circuit and piezoresistive effect, the relationship between the output voltages and the relative changes of piezoresistors can be expressed as follows [14]:

$$
\left\{\begin{array}{l}
V_{\text {out } x}=V_{x 1}-V_{x 2}=\frac{\Delta R_{x}}{R_{x 0}} \cdot V_{\mathrm{DD}} \\
V_{\text {out } y}=V_{y 1}-V_{y 2}=\frac{\Delta R_{y}}{R_{y 0}} \cdot V_{\mathrm{DD}} \\
V_{\text {out } z}=V_{z 1}-V_{z 2}=\frac{\Delta R_{z}}{R_{z 0}} \cdot V_{\mathrm{DD}}
\end{array}\right.
$$

where $R_{x 0}, R_{y 0}$ and $R_{z 0}$ are the piezoresistors under no acceleration, $V_{\text {out } x}, V_{\text {outy }}$ and $V_{\text {out } z}$ are the output voltages of $W_{1}, W_{2}$, and $W_{3}$, with the value of zero under the same condition.

In view of Equation (1), the output voltages of the Wheatstone bridges are directly proportional to the relative changes of the piezoresistors at a constant supply voltage. Thus, it is possible to measure the external acceleration based on the output voltage, where $\Delta R$ relative to stress is given in Equation (2) [15]:

$$
\frac{\Delta R}{R}=\pi_{l} \sigma_{l}+\pi_{t} \sigma_{t}
$$

where $\pi_{l}$ and $\pi_{t}$ are the longitudinal and transverse piezoresistance coefficients, and $\sigma_{l}$ and $\sigma_{t}$ are the longitudinal and transverse stress of the piezoresistors, respectively.

Due to the fact that the proposed accelerometer was designed on the SOI wafer with a device layer of n-type silicon with a $<100>$ orientation, through combining Equation (1) with Equation (2), the relationship between the output voltages and the stress can be expressed as Equation (3):

$$
\left\{\begin{array}{l}
V_{\text {out } x}=\frac{V_{\mathrm{DD}}}{2} \cdot \pi_{44}\left(\sigma_{l x}-\sigma_{t x}\right) \\
V_{\text {out } y}=\frac{V_{\mathrm{DD}}}{2} \cdot \pi_{44}\left(\sigma_{l y}-\sigma_{t y}\right) \\
V_{\text {out } z}=\frac{V_{\mathrm{DD}}}{2} \cdot \pi_{44}\left(\sigma_{l z}-\sigma_{t z}\right)
\end{array}\right.
$$

where $\sigma_{l x}$ and $\sigma_{t x}$ are the longitudinal and transverse stress of $R_{x 1}, R_{x 2}, R_{x 3}$ and $R_{x 4}, \sigma_{l y}$ and $\sigma_{\mathrm{t} y}$ are the longitudinal and transverse stress of $R_{y 1}, R_{y 2}, R_{y 3}$ and $R_{y 4}, \sigma_{l z}$ and $\sigma_{t z}$ are the longitudinal and transverse stress of $R_{z 1}, R_{z 2}, R_{z 3}$ and $R_{z 4}, \pi_{44}$ is piezoresistive coefficient.

According to the sensitivity definition and sensitive principle of the three-axis accelerometer, the output voltages of the Wheatstone bridges along three axes can be expressed as Equation (4):

$$
\left[\begin{array}{c}
V_{\text {out } x} \\
V_{\text {out } y} \\
V_{\text {out } z}
\end{array}\right]=\left[\begin{array}{c}
S_{x x} S_{x y} S_{x z} \\
S_{y x} S_{y y} S_{y z} \\
S_{z x} S_{z y} S_{z z}
\end{array}\right]\left[\begin{array}{c}
a_{x} \\
a_{y} \\
a_{z}
\end{array}\right]
$$

where $S_{x x}, S_{y y}$ and $S_{z z}$ are the sensitivities along the $x$-axis, $y$-axis and $z$-axis, respectively. $S_{x y}$ and $S_{x z}$ are the cross-axis sensitivities along the $x$-axis under $a_{y}$ and $a_{z}$, respectively. $S_{y x}$ and $S_{y z}$ are the cross-axis sensitivities along the $y$-axis under $a_{x}$ and $a_{z}$, respectively. $S_{z x}$ and $S_{z y}$ are the cross-axis sensitivities along the $z$-axis under $a_{x}$ and $a_{y}$, respectively.

In an ideal case, the elastic deformations of the four double L-shaped beams under the action of $a_{z}$ are identical, resulting in the same absolute variations of piezoresistors on the four double L-shaped beams, without the changes of the output voltages of $W_{1}$ and $W_{2}$ as well as the changes of the cross-axis sensitivities $\left(S_{x z}\right.$ and $S_{y z}$ ) of the sensor. Similarly, all the cross-axis sensitivities of $S_{y x}, S_{z x}, S_{z y}$ and $S_{x y}$ are zero and can be ignored. Thus, Equation (4) can be simplified as Equation (5):

$$
\left[\begin{array}{l}
V_{\text {out } x} \\
V_{\text {out } y} \\
V_{\text {out } z}
\end{array}\right]=\left[\begin{array}{ccc}
S_{x x} & 0 & 0 \\
0 & S_{y y} & 0 \\
0 & 0 & S_{z z}
\end{array}\right]\left[\begin{array}{l}
a_{x} \\
a_{y} \\
a_{z}
\end{array}\right]
$$


Based on the above theoretical analysis, it is possible to realize the measurement of acceleration along the $x$-axis, $y$-axis and $z$-axis by the accelerometer. Meanwhile, the cross-axis sensitivity can be ignored by structural optimization under the ideal process conditions and the ideal environmental testing conditions, etc.

\section{Simulation and Fabrication Technology}

\subsection{Simulation of Three-axis Accelerometer}

To study how $w$ affects the stress distribution, the finite element simulation software-Ansys 15.0 was used to simulate the characteristics of the proposed sensor, as shown in Figure 3. Figure 3a,b shows the top and bottom views of the sensor model with four double L-shaped beams, intermediate double beams and two mass blocks.
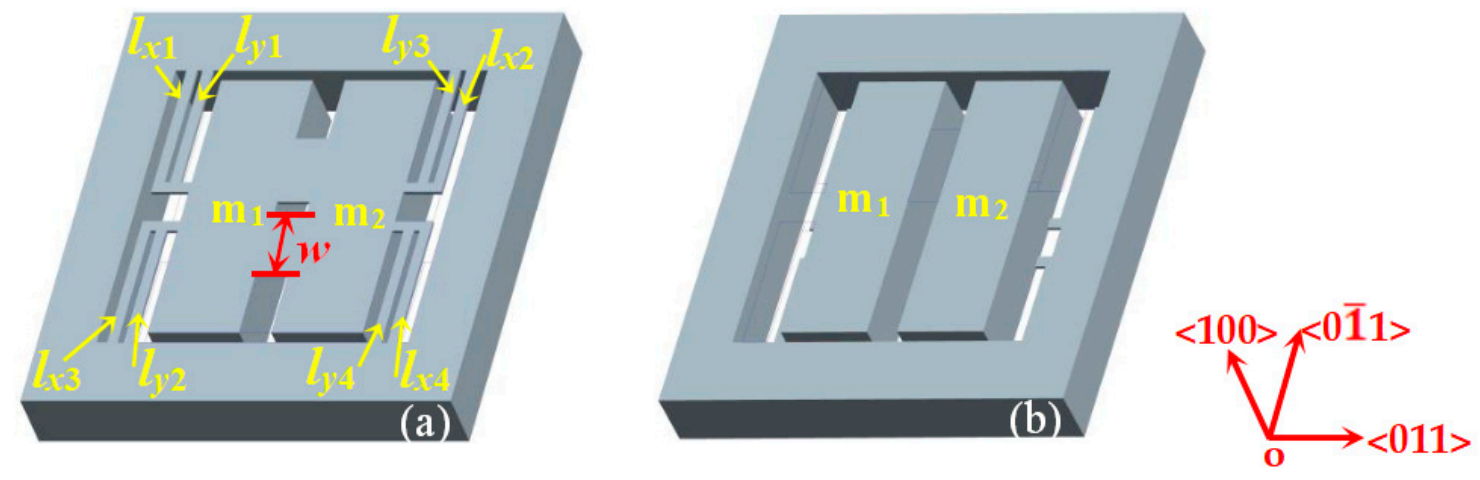

Figure 3. The structure model of the three-axis accelerometer: (a) top view; (b) bottom view.

On the basis of the model, the relationship between the average stress at the piezoresistor positions on the beams and $w$ under the actions of $a_{x}, a_{y}$ and $a_{z}$ was investigated. By respectively exerting an external acceleration of $15 \mathrm{~g}$ along the $x$-axis, $y$-axis and $z$-axis to the sensor model, six sensors (see Table 1) with different $w$ values were simulated. Then, the average stress at the corresponding piezoresistor positions was given, as shown in Figure 4. It can be seen that the average stress at the piezoresistor positions along teh $z$-axis decreases with the increase of $w$. With respect to that, the ones along the $x$-axis and $y$-axis do not change much, as shown in Figure 4. However, when increasing $w$ up to $500 \mu \mathrm{m}$, the average stress differences along the three axes are very small, and the measurement consistency is good. According to the derived equation in Section 2, it can be found that $S_{x x}, S_{y y}$ and $S_{z z}$ increase with the increase of the average stress, resulting in the decrease of the sensitivity along the $z$-axis with an increasing $w$. Finally, a better consistence of sensitivity is achieved when $w$ is $500 \mu \mathrm{m}$.

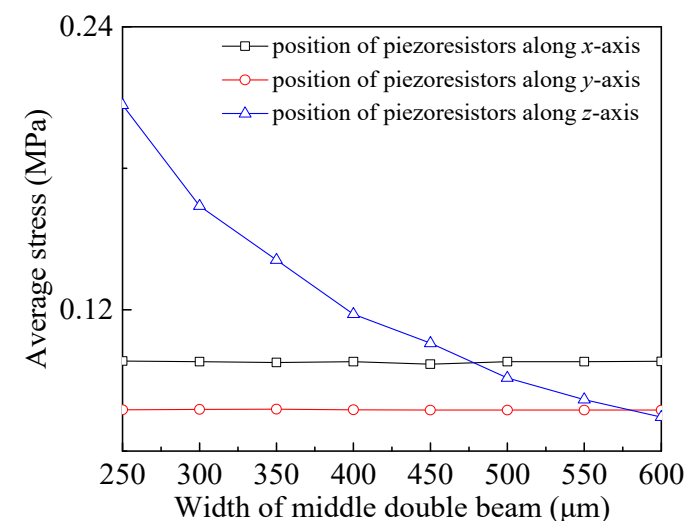

Figure 4. The relationship curves between the average stress at the piezoresistor positions along three axes and the width $(w)$ of the intermediate double beams. 


\subsection{The Fabrication Process of Three-axis Accelerometer}

As shown in Figure 5, the chip was fabricated on a SOI wafer with a n-type $<100>$ orientation device layer and resistivity of $0.1 \Omega \cdot \mathrm{cm}$ by using MEMS technology, in which the insets of mask1-mask7 used for photolithography and bonding glass are given together. The synthesis procedure is illustrated as follows:

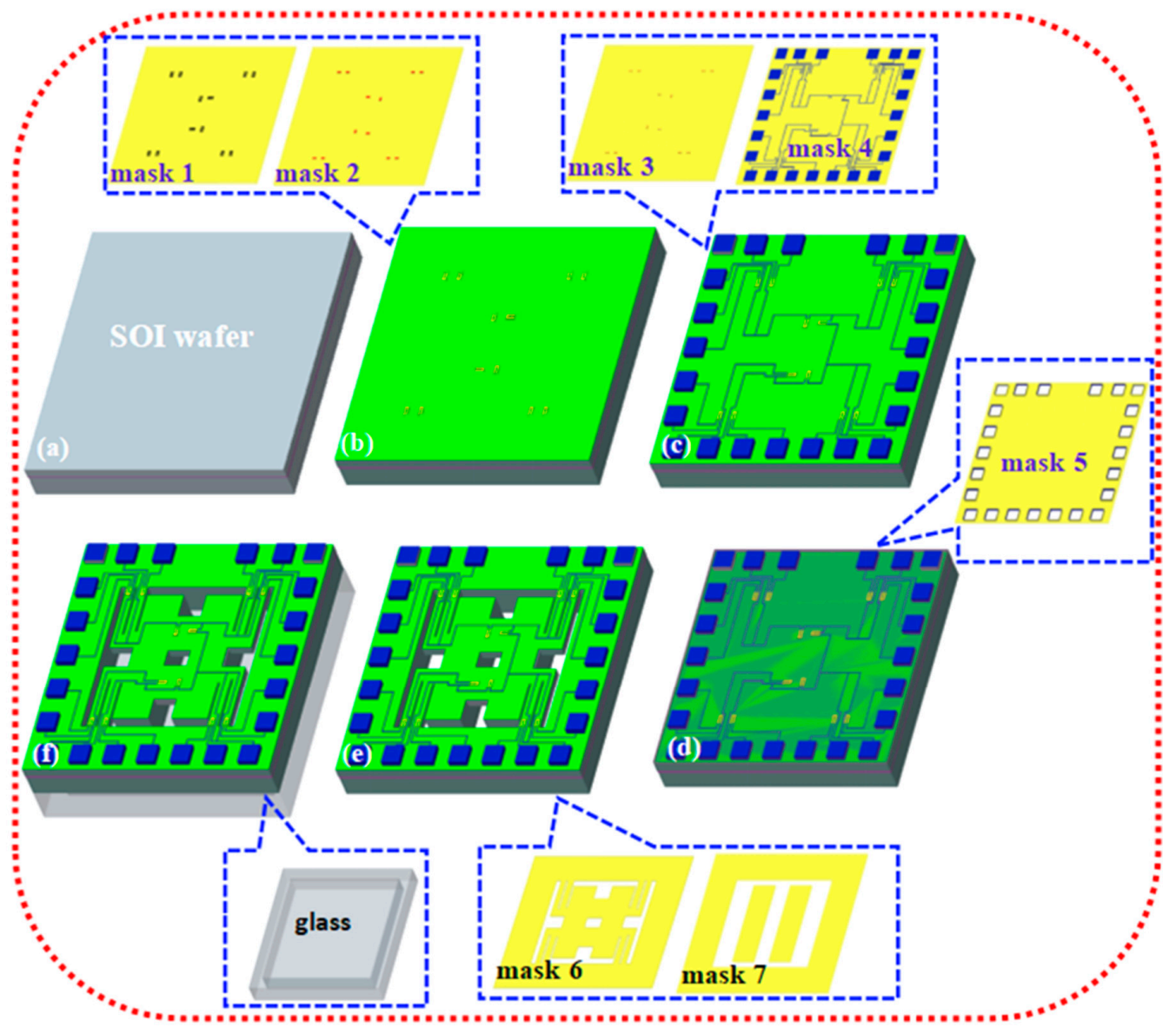

Figure 5. The main fabrication process of the proposed sensor (insets of mask1-mask7 used for photolithography and bonding glass): (a) cleaning the wafer; (b) forming $\mathrm{p}+$ and $\mathrm{p}$ - regions; (c) performing contact holes to fabricate the electrodes and interconnects; (d) forming pad; (e) ICP etching the top and bottom surfaces of the chip to release the beam structure; and (f) bonding a glass plate with the bottom surface of chip.

(a) Cleaning a SOI wafer using a standard cleaning method, then growing a $\mathrm{SiO}_{2}$ layer with a thickness of $50 \mathrm{~nm}$ by thermal oxidation method as an ion implantation buffer; (b) first, using photolithography to form an ion implantation window, performing the $\mathrm{p}+$ region by utilizing ion implantation, and secondly using photolithography to etch the windows of piezoresistors, performing the $\mathrm{p}$ - region as piezoresistors repeating the above method. After that, placing the chip in a vacuum environment of $1000{ }^{\circ} \mathrm{C}$ for 20 30 min to activate impurity ions so as to form an impurity distribution and effectively eliminate the damage caused by the ion implantation. Etching an $\mathrm{SiO}_{2}$ layer of $50 \mathrm{~nm}$ using wet etching technology, and thereafter growing the $\mathrm{SiO}_{2}$ layer as an insulating layer by using plasma enhanced chemical vapor deposition (PECVD); (c) third, photolithography to etch the top surface and performing contact holes between the piezoresistors and the Al electrodes, fabricating metal $\mathrm{Al}$ on the top surface by a vacuum evaporation method, and fourth, photolithography to form the electrodes and interconnects, and then metalizing at $420^{\circ} \mathrm{C}$ for $30 \mathrm{~min}$ to achieve ohmic contact; (d) growing a $\mathrm{SiO}_{2}$ layer by using PECVD as a passive layer to protect the $\mathrm{Al}$ electrode, and 
fifth, photolithography to form a pad; (e) etching the bottom surface of the chip by inductive couple plasma (ICP) technology [16] to form two mass blocks, thereafter etching the top surface of the chip by the identical technology to release four double L-shaped beams and intermediate double beams; (f) bonding a glass plate with a groove with the bottom surface of the chip by using electrostatic bonding technology to provide enough moving space for the two mass blocks.

To observe the morphology of the chip, a high-precision measuring microscope (Japan, Olympus, STM7) was used. Due to the large size $(4000 \mu \mathrm{m} \times 4000 \mu \mathrm{m})$ of the chip, the morphology stitching mode was used to complete the whole photograph. Figure $6 \mathrm{a}, \mathrm{b}$ shows the top and bottom photographs of the fabricated chip, respectively. As shown in Figure 6a, the potential labeling of pad points on the chip corresponds to that in the equivalent circuit in Figure 2. Meanwhile, using the Dow Corning fluorosilicone solvent-resistant sealant attached the chip to the package-printed circuit board (PCB) [17]. After fully curing the sealant, bonding the leads between the chip and PCB using an integrated chip bonding machine (American, KNS, 4526) realized the connection between the solder joints on the chip and the corresponding solder joints on the PCB, as shown in Figure 6c [14].

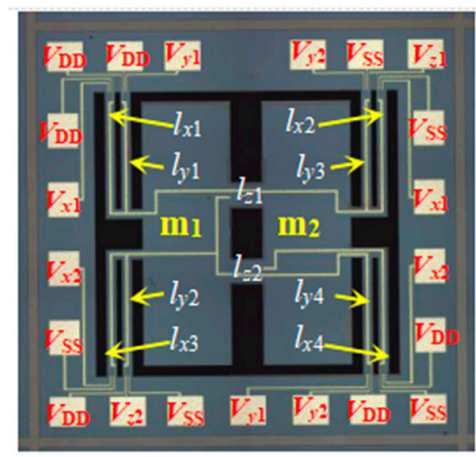

(a)

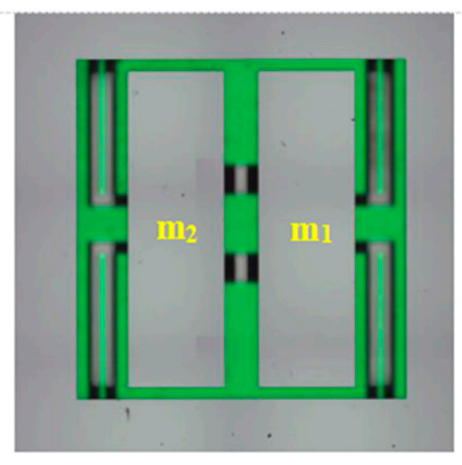

(b)

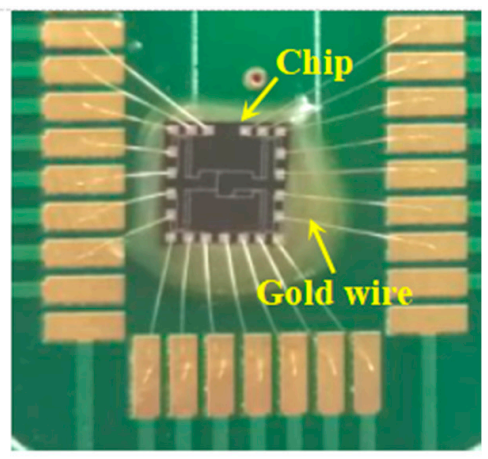

(c)

Figure 6. The photograph of the proposed chip: (a) top view; (b) bottom view; and (c) packaged chip.

\section{Results and Discussion}

To study the characteristics of the three-axis accelerometer, a testing system was built, consisting of a standard vibration table (China, Dongling, ESS-050) with an excitation frequency range of $0 \sim 10,000 \mathrm{~Hz}$ and acceleration of $0 \sim 30 \mathrm{~g}$ with an accuracy of $0.1 \mathrm{~g}$, a high performance digital multimeter (America, Agilent, 34410A), and a programmable linear direct-current power (China, Rigol, DP832A) to supply a voltage of $0 \sim 30 \mathrm{~V}$. The test was carried out at room temperature and a humidity of $40 \% \mathrm{RH}$.

\subsection{Frequency Response Characteristics}

To investigate the frequency response characteristics of the six types of sensors, a frequency response characteristic test was carried out under conditions of the supply voltage of $5.0 \mathrm{~V}$ and an external acceleration of $3 \mathrm{~g}$ with a frequency range from $100 \mathrm{~Hz}$ to $10,000 \mathrm{~Hz}$. Through taking the chip with $w=500 \mu \mathrm{m}$ as an example, the characteristic curves of the resonance frequency along the $x$-axis, $y$-axis and $z$-axis are respectively shown in Figure 7a-c. As shown in Figure 7a, the test result shows that the output voltages of the sensor do not markedly change with an increase of the excitation frequency. When increasing the frequency of the excitation signal up to $8898 \mathrm{~Hz}$ along the $x$-axis, the output voltage of the sensor along the $x$-axis reaches a maximum. Then, the output voltage of the sensor begins to gradually decrease as the vibration frequency continually increases, thus achieving the resonance frequency of $8898 \mathrm{~Hz}$ along the $x$-axis when $w=500 \mu \mathrm{m}$. Similarly, when $w$ is $500 \mu \mathrm{m}$ the resonance frequencies along the $y$-axis and $z$-axis are $8395 \mathrm{~Hz}$ and $3270 \mathrm{~Hz}$, as shown in Figure $7 \mathrm{~b}, \mathrm{c}$. After that, a wide frequency sweep was performed on the proposed sensor, i.e., with a vibration frequency range from $100 \mathrm{~Hz}$ to $10,000 \mathrm{~Hz}$ at constant acceleration. The resonance frequencies of the 
six types of chips (named as AS-1 AS-6, corresponding to different $w$ values of 250-500 $\mu \mathrm{m}$ ) were obtained by observing the output voltage changes, as shown in Table 2. According to Table 2, due to the fact that the two identical mass blocks in the basic structure of the accelerometer were connected by the intermediate double beams, a collective movement can be caused to form the deformations of the double L-shaped beams, benefitting the measurement of $a_{x}$ or $a_{y}$. However, the widths of the intermediate double beams $(w)$ have little influence on the movement along the $x$-axis or $y$-axis, without monotonous changes of the resonance frequency along the $x$-axis and $y$-axis with $w$.

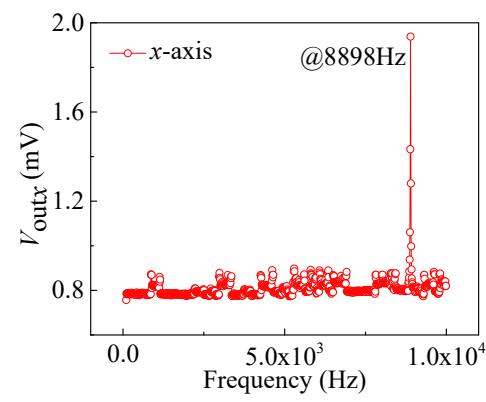

(a)

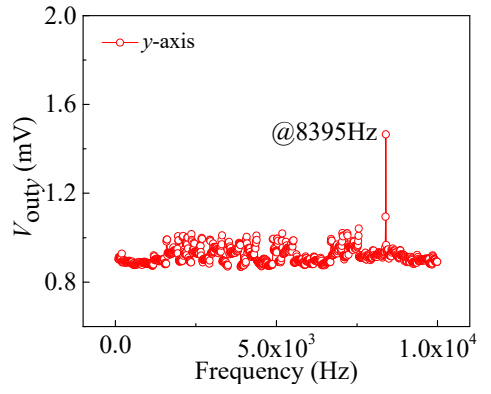

(b)

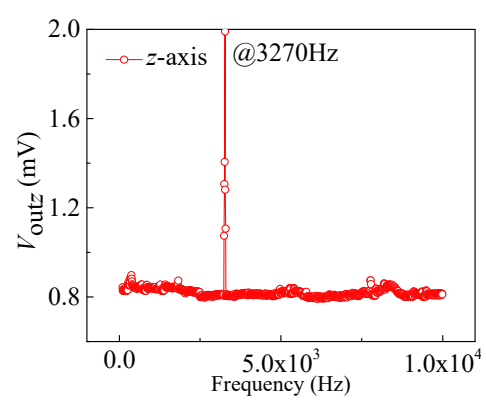

(c)

Figure 7. The characteristic curves of resonance frequency when $w$ is $500 \mu \mathrm{m}$ : (a) along the $x$-axis; (b) along the $y$-axis; and (c) along the z-axis.

Table 2. The resonance frequencies of the proposed sensor with different intermediate double beam widths.

\begin{tabular}{ccccc}
\hline Type & $\boldsymbol{w}(\boldsymbol{\mu \mathrm { m } )}$ & $\begin{array}{c}\text { Resonance } \\
\text { Frequency } \\
\text { along } \boldsymbol{x} \text {-axis (Hz) }\end{array}$ & $\begin{array}{c}\text { Resonance } \\
\text { Frequency } \\
\text { along } \boldsymbol{y} \text {-axis (Hz) }\end{array}$ & $\begin{array}{c}\text { Resonance } \\
\text { Frequency } \\
\text { along } \boldsymbol{z} \text {-axis (Hz) }\end{array}$ \\
\hline AS-1 & 250 & 9106 & 8956 & 3211 \\
AS-2 & 300 & 9374 & 7963 & 3221 \\
AS-3 & 350 & 7787 & 8957 & 3314 \\
AS-4 & 400 & 8692 & 8694 & 3250 \\
AS-5 & 450 & 7996 & 8348 & 3109 \\
AS-6 & 500 & 8898 & 8395 & 3270 \\
\hline
\end{tabular}

\subsection{Sensitivity and Cross Interference Characteristics}

To research the sensitivity characteristics of the six types of sensors, the sensitivity test was repeated three times under the conditions of a supply voltage of $5.0 \mathrm{~V}$ and acceleration along the sensitive axis from $0 \mathrm{~g}$ to $15 \mathrm{~g}$ with a step of $3 \mathrm{~g}$. Figure 8 gives the relationship curves between the output voltages and the external acceleration along the three axes, where $V_{\text {out } x}$ (see the black line), $V_{\text {outy }}$ (see the red line) and $V_{\text {outz }}$ (see the blue line) are the output voltages for the resulting sensors. As shown in Figure 8a, it can be seen that the output voltages $V_{\text {out } x}$ and $V_{\text {outy }}$ of the AS- 1 sensor linearly increase with an increase of $a_{x}$ and $a_{y}$, respectively. With respect to that, the output voltage $V_{\text {outz }}$ of the sensor nonlinearly increases with an increase of $a_{z}$. In addition, when $a_{x}=a_{y}=a_{z}, V_{\text {outz }}$ are markedly bigger than $V_{\text {outx }}$ and $V_{\text {out } y}$, where $V_{\text {outy } y}$ is close to $V_{\text {out } x}$. This indicates that the resulting AS-1 sensor can realize the acceleration measurement along the three axes, but with a low linearity and higher sensitivity along the $z$-axis when compared with the other axes. As shown in Figure 8b-e, the sensors of AS-2, AS-3, AS-4 and AS-5, when compared with AS-1, have similar sensitivities and linearity along the three axes. In particular, the corresponding output voltages of the AS-6 sensor linearly increase, not only with the increasing of the external accelerations $a_{x}$ and $a_{y}$, but also with the increasing of $a_{z}$, as shown in Figure $8 \mathrm{f}$. In addition, when $a_{x}=a_{y}=a_{z}, V_{\text {out } x}, V_{\text {outy }}$ and $V_{\text {outz }}$ are close, all having a relationship curve with an approximate linearity. On the basis of this, the characteristics of the AS-6 sensor are in accord with the previous simulating results. 

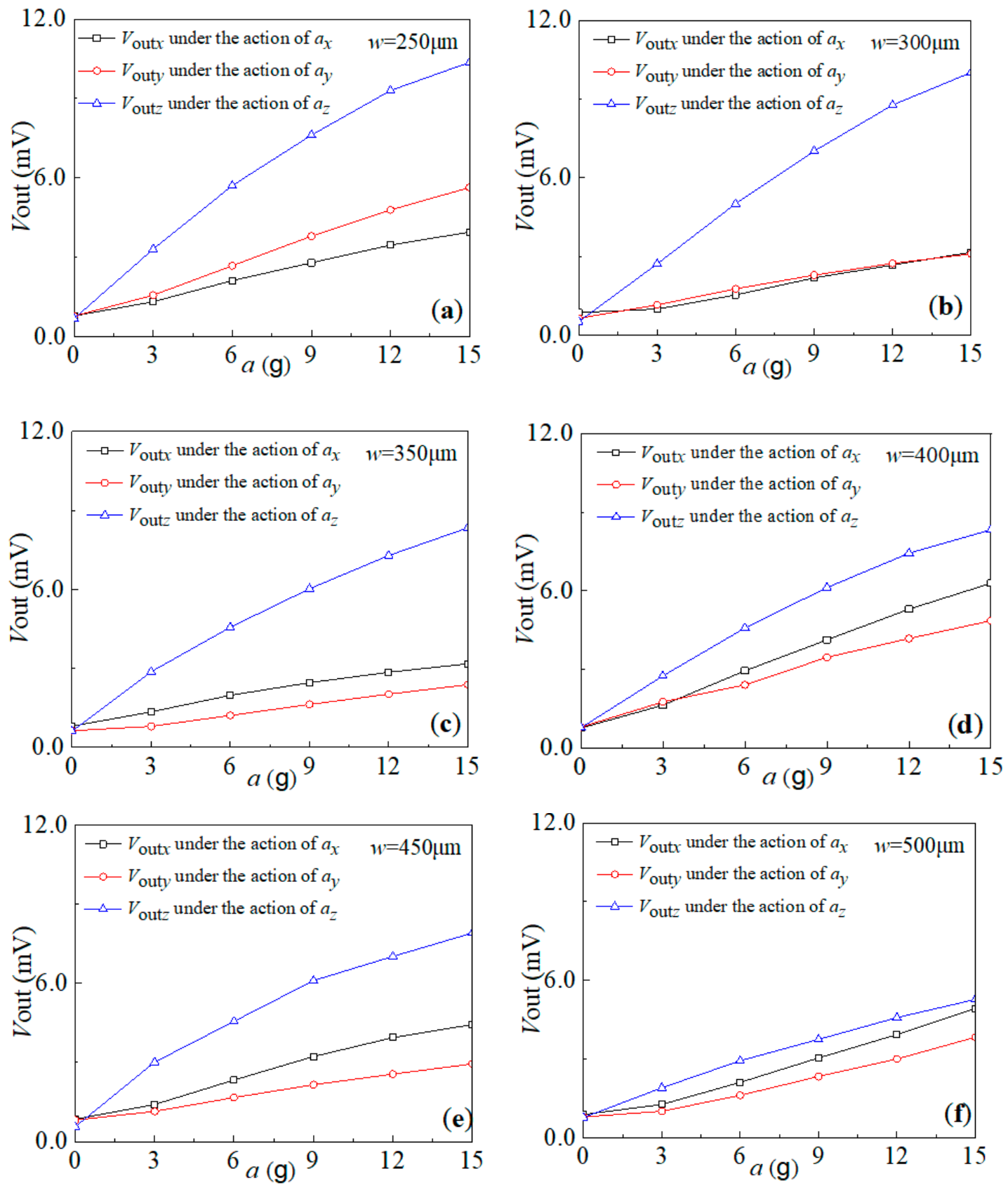

Figure 8. The relationship curves between the output voltages of the proposed sensor and the external acceleration of the six types of sensors with different $w$ values: (a) $w=250 \mu \mathrm{m}$; (b) $w=300 \mu \mathrm{m}$; (c) $w=350 \mu \mathrm{m} ;(\mathbf{d}) w=400 \mu \mathrm{m} ;(\mathbf{e}) w=450 \mu \mathrm{m}$; and (f) $w=500 \mu \mathrm{m}$.

In addition, according to the sensitivity definition and testing data, the sensitivities of the proposed sensor with different $w$ values are shown in Table 3. It indicates that the sensitivities of three-axis accelerometers decrease along the $z$-axis with the increasing widths of the intermediate double beams. When $w$ is $500 \mu \mathrm{m}$, the proposed sensor achieves a better consistence of sensitivities along the three axes, with sensitivities of $0.302 \mathrm{mV} / \mathrm{g}, 0.235 \mathrm{mV} / \mathrm{g}$ and $0.347 \mathrm{mV} / \mathrm{g}$, respectively. 
Table 3. The sensitivities of the proposed sensor with different widths of intermediate double beams.

\begin{tabular}{cccccccc}
\hline Type & $w(\mu \mathrm{m})$ & $S_{x x}(\mathbf{m V} / \mathbf{g})$ & $\begin{array}{c}S_{y y} \\
(\mathbf{m V} / \mathbf{g})\end{array}$ & $S_{z z}(\mathbf{m V} / \mathbf{g})$ & $\begin{array}{c}\text { Linearity } \\
(x \text {-axis })\end{array}$ & $\begin{array}{c}\text { Linearity } \\
(\boldsymbol{y} \text {-axis) }\end{array}$ & $\begin{array}{c}\text { Linearity } \\
(z \text {-axis })\end{array}$ \\
\hline AS-1 & 250 & 0.211 & 0.324 & 0.646 & $3.3 \%$ & $2.7 \%$ & $9.1 \%$ \\
AS-2 & 300 & 0.135 & 0.163 & 0.633 & $7.6 \%$ & $5.4 \%$ & $6.8 \%$ \\
AS-3 & 350 & 0.158 & 0.117 & 0.515 & $7.1 \%$ & $5.5 \%$ & $7.2 \%$ \\
AS-4 & 400 & 0.369 & 0.271 & 0.504 & $3.0 \%$ & $3.2 \%$ & $8.3 \%$ \\
AS-5 & 450 & 0.239 & 0.142 & 0.470 & $5.3 \%$ & $3.5 \%$ & $10.0 \%$ \\
AS-6 & 500 & 0.302 & 0.235 & 0.347 & $4.1 \%$ & $3.4 \%$ & $3.4 \%$ \\
\hline
\end{tabular}

To further study the cross-interference characteristics of the sensitivity of the AS-6 sensor, the output voltages along the three axes at constant accelerations of $a_{x}, a_{y}$ and $a_{z}$ were investigated at the same time, respectively. Figure 9 shows the relationship curves between the output voltages and the corresponding acceleration. As shown in Figure $9 a, V_{\text {out } x}$ along the sensitive axis linearly increases with the increasing $a_{x}$, but with slight changes in $V_{\text {outy }}$ and $V_{\text {outz }}$ under the same acceleration, respectively. This indicates that the $V_{\text {outy }}$ and $V_{\text {outz }}$ of the cross-interference caused by $a_{x}$ are low. Similarly, the output voltage $V_{\text {outy }}$ (or $V_{\text {outz }}$ ) linearly increases with the increasing $a_{y}$ (or $a_{z}$ ), with slight changes in the other output voltages under the action of $a_{y}\left(\right.$ or $\left.a_{z}\right)$, indicating that the $V_{\text {out } x}$ and $V_{\text {outz }}$ (or $V_{\text {out } x}$ and $V_{\text {outy }}$ ) of the cross-interference caused by $a_{y}$ (or $a_{z}$ ) are small as well, as shown in Figure $9 b, c$. The test result indicates that it is possible to achieve a high output voltage along the sensitive axis and a low cross-interference along the non-sensitive axes using the AS-6 sensor.
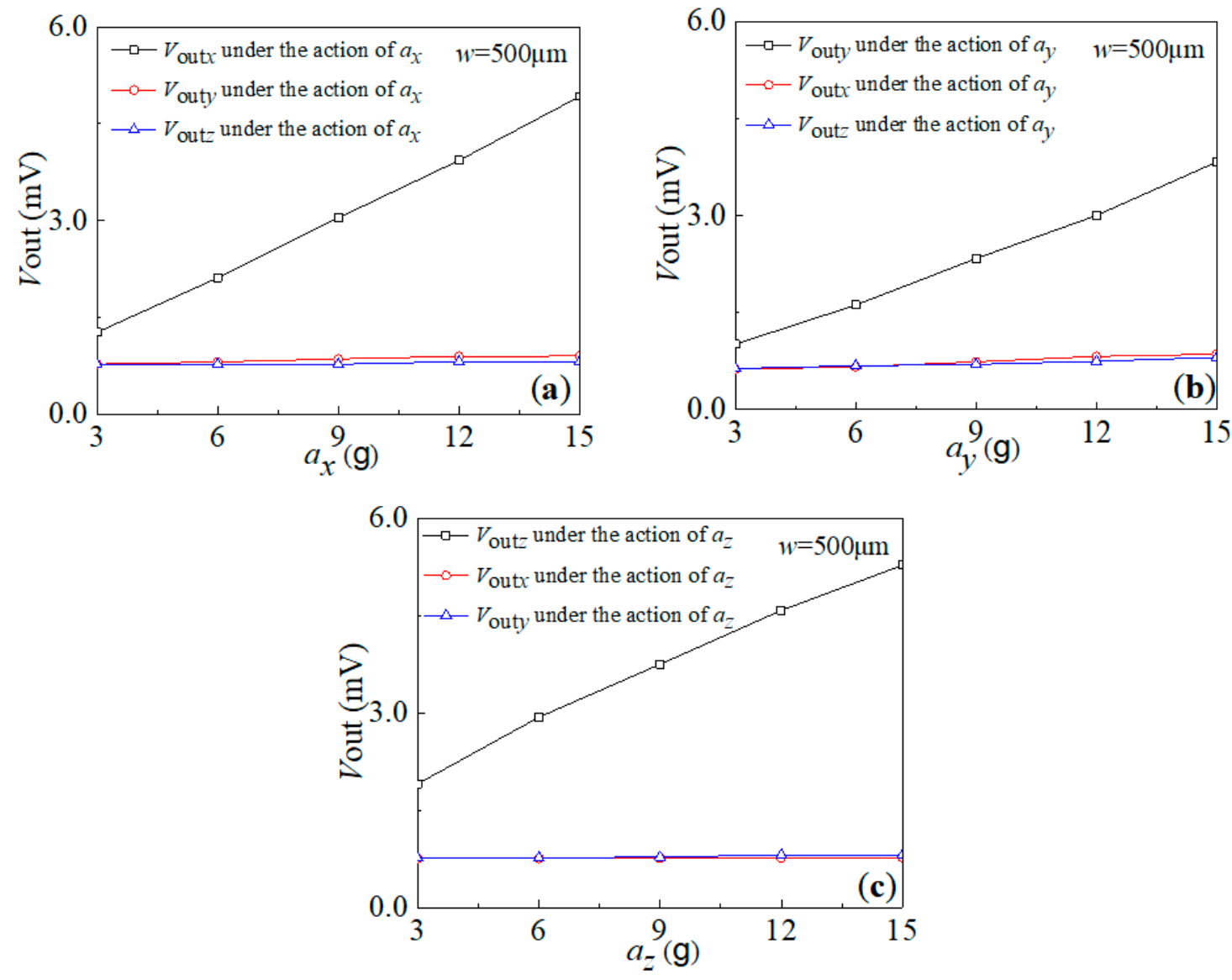

Figure 9. The cross-interference characteristic curves of the AS-6 sensor under three-axis acceleration along: (a) $x$-axis; (b) $y$-axis; (c) $z$-axis. 
In addition, the characteristic parameters of the AS- 6 sensor are shown in Table 4. It can be seen that, when $w$ is $500 \mu \mathrm{m}$, maximum and minimum cross-axis sensitivities can be achieved by the proposed sensor, i.e., $0.019 \mathrm{mV} / \mathrm{g}$ and $0.001 \mathrm{mV} / \mathrm{g}$, respectively. According to the above data analysis, it is possible to improve the consistency of sensor sensitivity and reduce the cross-interference by using the proposed scheme.

Table 4. The characteristic parameters of the AS-6 sensor.

\begin{tabular}{cccccc}
\hline Characteristic Parameters & $\begin{array}{c}\text { Resonant } \\
\text { Frequency } \\
\text { (Hz) }\end{array}$ & $\begin{array}{c}\text { Bandwidth } \\
\mathbf{( H z )}\end{array}$ & & \multicolumn{3}{c}{$\begin{array}{c}\text { Sensitivity of the Sensor along the } \boldsymbol{x} \text {-axis, } \\
\boldsymbol{y} \text {-axis and } z \text {-axis } \\
\text { under Resonant Frequency } \mathbf{( m V / g )}\end{array}$} \\
\cline { 4 - 6 } Acceleration Sensor & 8898 & $100-8000$ & 0.302 & 0.011 & 0.004 \\
\hline Sensor along $x$ axis & 8395 & $100-7500$ & 0.019 & 0.235 & 0.014 \\
Sensor along $y$ axis & 3270 & $100-3000$ & 0.001 & 0.004 & 0.347 \\
Sensor along $z$ axis & &
\end{tabular}

\section{Conclusions}

In summary, a three-axis accelerometer with double L-shaped beams was designed and fabricated using micro-electromechanical systems (MEMS) technology in this study. When applying acceleration to the proposed sensor chip, the corresponding acceleration along the three axes could be measured according to the output voltage changes of three Wheatstone bridges based on the elastic force theory and piezoresistive effect. To investigate the characteristics of the accelerometer, the effects of the width of intermediate double beams on the characteristics were simulated to optimize the structure size. Based on that, six types of sensors with different sizes were fabricated on silicon-on-insulator (SOI) wafers using MEMS technology and were packaged on printed circuit boards (PCB) using an electrostatic bonding process and inner lead bonding technology. The test results indicate that, at room temperature and $V_{\mathrm{DD}}=5.0 \mathrm{~V}$, better sensitivities along the three axes can be achieved by the resulting sensor with an optimized size, i.e., $0.302 \mathrm{mV} / \mathrm{g}, 0.235 \mathrm{mV} / \mathrm{g}$ and $0.347 \mathrm{mV} / \mathrm{g}$, respectively. This study provides an effective fabrication method to further improve the sensitivity characteristics of three-axis accelerometers.

Author Contributions: X.Z. and D.W. conceived and designed the experiments; X.Z. and Y.W. performed the simulations and experiments; X.Z. and Y.W. analyzed the data; and X.Z. and Y.W. wrote the paper. The remaining part of the work has been done together. All authors have read and agreed to the published version of the manuscript.

Funding: This research was funded by the National Natural Science Foundation of China (Grant No. 61971180, $61471159,61006057)$.

Conflicts of Interest: The authors declare no conflicts of interest

\section{References}

1. Varanis, M.; Silva, A.; Mereles, A.; Pederiva, R. MEMS accelerometers for mechanical vibrations analysis: A comprehensive review with applications. J. Braz. Soc. Mech. Sci. Eng. 2018, 11, 527. [CrossRef]

2. Balfas, M.; Ahamed, S.I.; Tamma, C.; Arif, M.; Kattan, A.J.; Chu, W. A Study and Estimation a Lost Person Behavior in Crowded Areas Using Accelerometer Data from Smartphones. In Proceedings of the 2018 IEEE 42nd Annual Computer Software and Applications Conference, Tokyo, Japan, 23-27 July 2018.

3. Kapti, A.O.; Muhurcu, G. Wearable acceleration sensor application in unilateral trans-tibial amputation prostheses. Biocybern. Biomed. Eng. 2014, 34, 53-62. [CrossRef]

4. Marco, M.; James, N.; Chrysovalantis, P. Mechanical structural design of a MEMS-based piezoresistive accelerometer for head injuries monitoring: A computational analysis by increments of the sensor mass moment of inertia. Sensors 2018, 18, 289.

5. Theresa, L.; Oliver, G.; Alexander, B. Using MEMS acceleration sensors for monitoring blade tip movement of wind turbines. In Proceedings of the 2018 IEEE SENSORS, New Delhi, India, 28-31 October 2018. 
6. Solouk, M.R.; Shojaeefard, M.H.; Dahmarde, M. Parametric topology optimization of a MEMS gyroscope for automotive applications. Mech. Syst. Signal Process. 2019, 128, 389-404. [CrossRef]

7. Yang, B.; Xi, J.; Yang, J.; Xue, L. An alignment method for strapdown inertial navigation systems assisted by doppler radar on a vehicle-borne moving base. Sensors 2019, 19, 4577. [CrossRef] [PubMed]

8. Mohankumar, P.; Ajayan, J.; Yasodharan, R.; Devendran, P.; Sambasivam, R. A review of micromachined sensors for automotive applications. Measurement 2019, 140, 305-322. [CrossRef]

9. Sankar, A.R.; Das, S. A very-low cross-axis sensitivity piezoresistive accelerometer with an electroplated gold layer atop a thickness reduced proof mass. Sens. Actuators A Phys. 2013, 189, 125-133. [CrossRef]

10. Han, J.; Zhao, Z.; Niu, W.; Huang, R.; Dong, L. A low cross-axis sensitivity piezoresistive accelerometer fabricated by masked-maskless wet etching. Sens. Actuators A Phys. 2018, 283, 17-25. [CrossRef]

11. Takahashi, H.; Kan, T.; Nakai, A.; Takahata, T.; Usami, T.; Shimoyama, I. Highly sensitive and low-crosstalk angular acceleration sensor using mirror-symmetric liquid ring channels and MEMS piezoresistive cantilevers. Sens. Actuators A Phys. 2019, 287, 39-47. [CrossRef]

12. Bakhoum, E.G.; Cheng, M.H.M.; Kyle, R.A. 3-Axis, ultrahigh-sensitivity, miniature acceleration sensor. IEEE Trans. Compon. Packag. Manuf. Technol. 2018, 8, 244-250. [CrossRef]

13. Zhao, X.; Li, S.; Ai, C.; Liu, H.; Wen, D. Fabrication and characterization of the Li-Doped ZnO thin films piezoelectric energy harvester with multi-resonant frequencies. Micromachines 2019, 10, 212. [CrossRef] [PubMed]

14. Zhao, X.; Wen, D.; Li, G. Fabrication and characteristics of the nc-Si/c-Si heterojunction MOSFETs pressure sensor. Sensors 2012, 12, 6369-6379. [CrossRef] [PubMed]

15. Singh, R.; Ngo, L.L.; Seng, H.S.; Mok, F.N.C. A silicon piezoresistive pressure sensor. In Proceedings of the First IEEE International Workshop on Electronic Design, Test and Applications '2002, Christchurch, New Zealand, 29-31 January 2002.

16. Munro, D. DIY MEMS; Springer: Berlin, Germany, 2019; pp. 31-47.

17. Dong, Y.; Min, X.; Kim, W.S. A 3D-printed integrated PCB-based electochemical sensor system. IEEE Sens. J. 2018, 18, 2959-2966. [CrossRef] 